\title{
Non-Parametric Analysis of Inter-Individual Relations Using an Attention-Based Neural Network
}

\author{
Takashi Morita*1, Aru Toyoda ${ }^{2}$, Seitaro Aisu ${ }^{1}$, Akihisa Kaneko ${ }^{1}$, Naoko Suda-Hashimoto ${ }^{1}$, \\ Ikki Matsuda $\mathrm{a}^{2,3,4,5}$, and Hiroki Koda ${ }^{1}$ \\ ${ }^{1}$ Primate Research Institute, Kyoto University \\ ${ }^{2}$ Chubu University Academy of Emerging Sciences \\ ${ }^{3}$ Wildlife Research Center of Kyoto University \\ ${ }^{4}$ Japan Monkey Centre \\ ${ }^{5}$ Institute for Tropical Biology and Conservation, Universiti Malaysia Sabah
}

December 7, 2020

\begin{abstract}
1. Social network analysis, widely adopted in animal studies over the past decade, enabled the revelation of global characteristic patterns of animal social systems from pairwise inter-individual relations.

2. Animal social networks are typically drawn based on geometric proximity and/or frequency of social behaviors (e.g., grooming), but the appropriate metric for inter-individual relatedness is not clear especially when prior knowledge on the species/data is limited.

3. In this study, the researchers explored a non-parametric analysis of inter-individual relations using a neural network with the attention mechanism, which plays a central role in natural language processing.

4. The high interpretability of the attention mechanism and the flexibility of the entire neural network allow automatic detection of inter-individual relations included in raw data, without requiring prior knowledge/assumptions about what modes/types of relations are included in the data.

5. For these case studies, three-dimensional location data collected from simulated agents and real Japanese macaques were analyzed.

6. The proposed method successfully recovered the latent relations behind the simulated data and discovered female-oriented relations in the real data that are in accordance with previous generalizations about macaque social structure.
\end{abstract}

Keywords: interaction, social network analysis, animal society, Japanese macaque, deep learning, attention mechanism

\section{Introduction}

Understanding the characteristics of animal social groups is a major objective of animal ecology. Complex social systems of eusocial insects and non-human primates are often viewed as biological models or precursors

\footnotetext{
${ }^{*}$ Corresponding author: tmorita@alum.mit.edu
} 
of human societies, and as such, have attracted the interest of social scientists as well. Social network analysis, widely adopted in animal studies over the past decade, can be used to determine global characteristic patterns of animal social systems from pairwise inter-individual relations (Krause et al., 2015).

The application of social network analysis to animal studies requires an appropriate metric for the pairwise relatedness between individuals (Sosa et al., 2020). Popular options of the relation metric include proximity, simply defined by geometric closeness between individuals (often thresholded and converted to counts of co-appearance within the threshold; Silk et al., 2006b,a; Croft et al., 2008; Clark, 2011; Boyland et al., 2013; Castles et al., 2014; Krause et al., 2015; Schofield et al., 2019; Gelardi et al., 2020), and frequency of social behaviors (e.g., grooming of non-human primates; Chepko-Sade et al., 1989; Silk et al., 2006b,a; Croft et al., 2008; Clark, 2011; King et al., 2011b,a; Castles et al., 2014; Krause et al., 2015). However, the appropriate relation metric is not necessarily clear, especially when prior knowledge about the species/data is limited (cf. Haddadi et al., 2011; Castles et al., 2014; Farine and Whitehead, 2015; Canteloup et al., 2020). For example, location data for measuring geometric proximity is available for a wide variety of animals. This includes even those species whose social activities are not yet well-known. Location information has also become more reliable and scalable thanks to recent developments in bio-logging technology, such as GPS (Heupel et al., 2006; Rutz and Hays, 2009; Rutz et al., 2012; Fehlmann and King, 2016; King et al., 2018; Dore et al., 2020; Gelardi et al., 2020). However, naively measuring the Euclidean distance between individuals and treating pairs with smaller distance as socially related is not necessarily reasonable; some animal agents, for instance, may follow other individuals while keeping a certain geometric distance (perhaps to avoid being attacked), but such distant relationship/dependency would be missed if only proximal co-occurrences are counted as the index. Distance could also have different social meanings if other factors, such as trees and other objects, intervene (Pays et al., 2007). Further, vertical divergences are expected to be less or not relevant in analysis of terrestrial animals, which do not fly or dive and so cannot control their vertical distance from other individuals.

Given the limitations of the conventional analytical paradigm depending on manually selected relation metrics, the present study explores a non-parametric, data-driven analyzer of inter-individual relations. Unlike the conventional analysis, the proposed approach automatically detects inter-individual relations from raw data using a machine learning technique (Figure 1; cf. Maekawa et al., 2020). For example, location logs of animals collected through bio-logging devices can be analyzed without the need to first determine relevant aspects of the data such as co-appearance within a certain distance. The detected relations can be used in additional analyses, including the modes/types of relations and social networks.

The proposed non-parametric analysis of inter-individual relations is based on an artificial neural network, which is the canonical framework in today's machine learning. Neural networks are universal approximators 


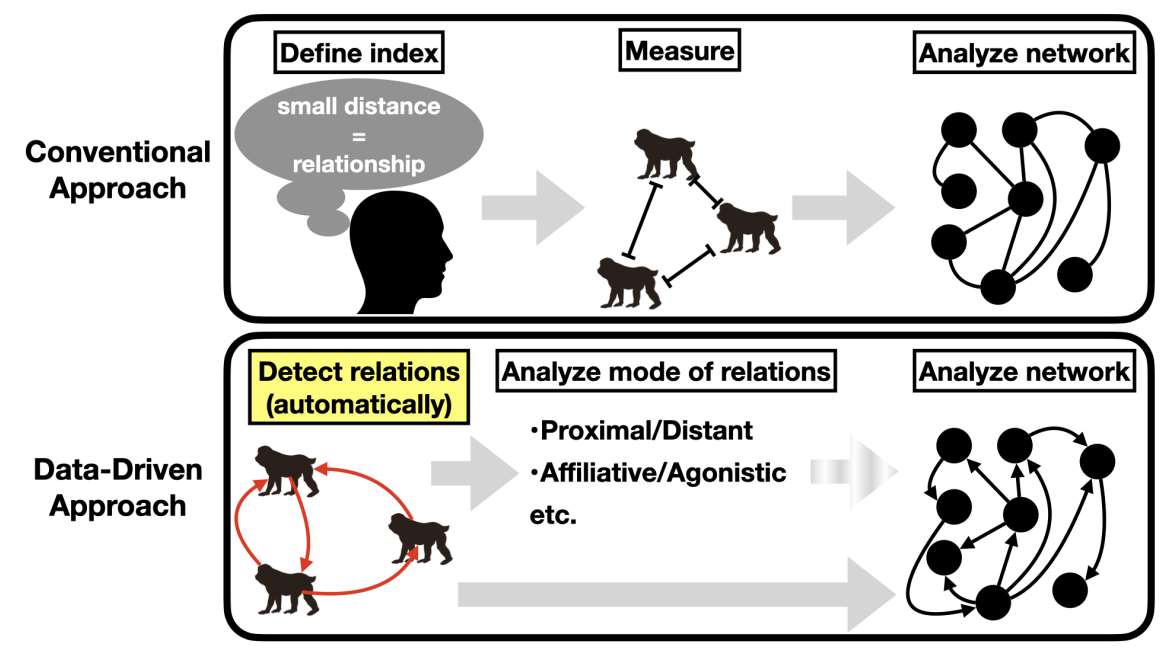

Figure 1: Comparison of the conventional approach to analysis of animal social relations/systems (top) with the alternative data-driven approach proposed in the current study (bottom). In the conventional approach, researchers first need to define an appropriate index for measuring relatedness between individual animal agents, which can be difficult for understudied animals. By contrast, the proposed approach first detects inter-individual relations automatically using machine learning techniques, without manual specification of relevant aspects of the data, and then uses the detected relations to further characterize the modes/types of relations and more global patterns via social network analysis. The present study proposes a method for the first step in the data-driven approach (highlighted in yellow).

of mathematical functions (i.e., they can approximate any types of computable functions, if ideally tuned; Cybenko, 1989; Hornik, 1991; Jin et al., 1995; Pérez et al., 2019) and thus can be used for analysis of a broad range of data without the assumptions about random distributions and latent structures behind the data. One drawback of using neural networks, however, is that they are often non-interpretable; neural network computation consists of thousands of summation, multiplication, non-linear activation (e.g., sigmoid and tanh), and other basic calculations, and does not explain what aspects/portions of data are important. To perform interpretable analysis of inter-individual relations, the present study exploits a special neural network module called the attention mechanism. The attention mechanism was originally invented for machine translation to represent relations between words in the source and target languages (Figure 2a; Bahdanau et al., 2015). More recently, studies have used the attention mechanism to represent word relations within input sentences (self-attention, Figure 2b; Vaswani et al., 2017; Devlin et al., 2018). It is now considered central technology in today's natural language processing (NLP). The present study applies this technology to reveal latent relations among animal agents that are reflected in their location data, without prior assumptions about the possible types of relation structures or noise distributions (Figure 2c; see Yuan and Jia, 2019; Maekawa et al., 2020, for other applications to biological studies). While only the location data are discussed in this study, there are many possible applications of our relation analysis given the flexibility of the neural network. 


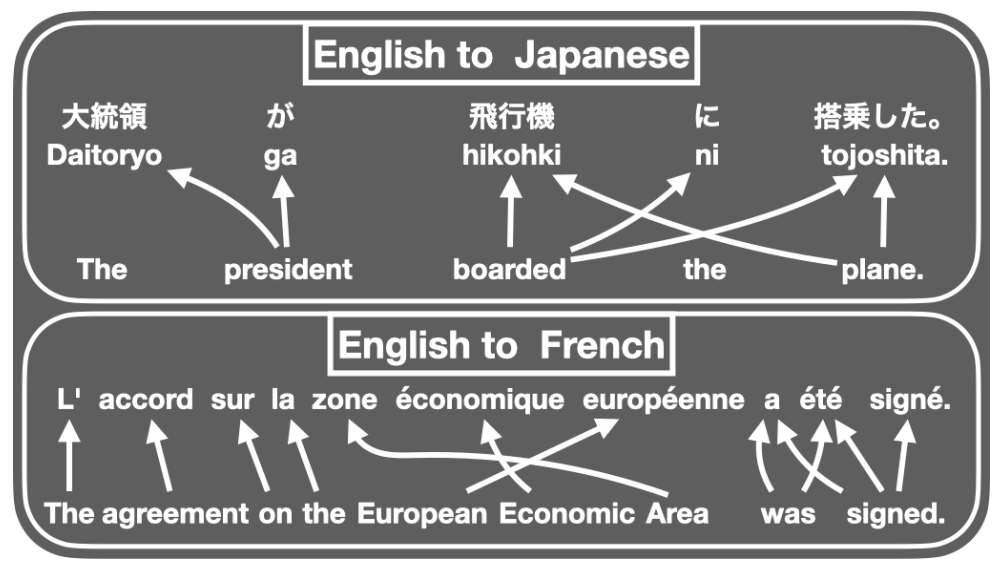

(a) Machine Translation

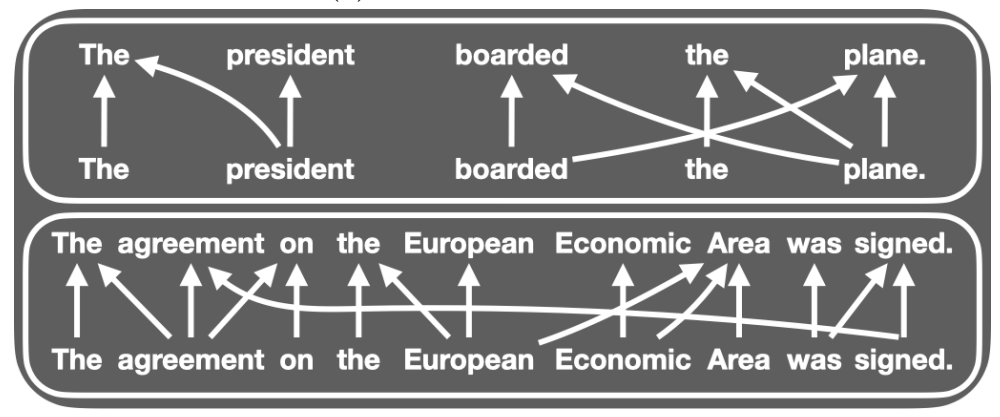

(b) Self-Attention

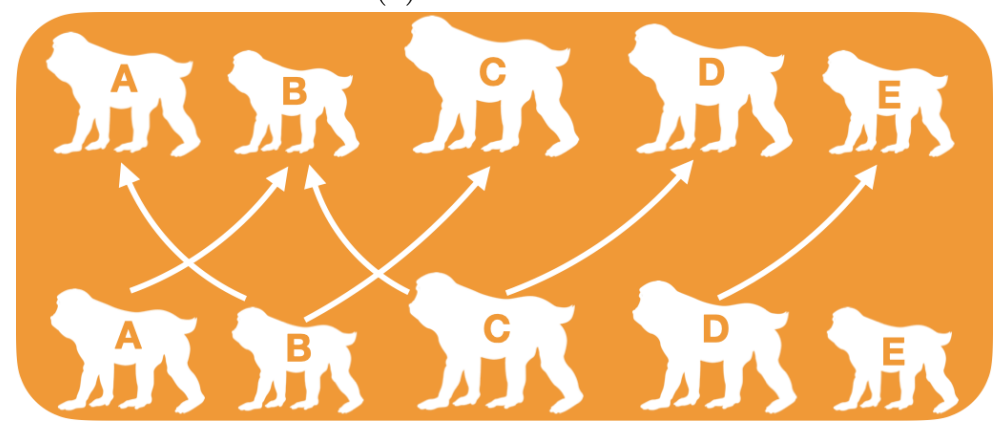

(c) Inter-Individual Relations

Figure 2: History of the use of the attention mechanism. (a) The original use of the attention mechanism in machine translation, relating words in the source language (English) to ones in the target language (Japanese/French). (b) More recent application in NLP that represents relations between words within the input sentences. (c) Application to inter-individual relations explored in this study. 
The proposed method is explored using three-dimensional (3D) location data collected from Japanese macaques (Macaca fuscata) as well as simulation data that clarify its advantages over the conventional distance-based analysis. Japanese macaques show stable relationships structured on dominance and kinship, and they have been studied extensively for more than 50 years as a way to understand the evolution of social systems in primate lineages, including humans (Nakagawa et al., 2010). This large accumulation of knowledge and data made the Japanese macaque an ideal species for evaluating our novel analysis. We note that the method is not limited to this species or primates generally, and could even be more valuable for studying animals that do not show interpretable social activities.

\section{Materials \& Methods}

\subsection{Attention-Based Analysis of Inter-Individual Relations}

This section provides an overview of the proposed methods in $\S 2.1 .1$ followed by a more detailed explanation in $\S 2.1 .2-2.1 .3$.

\subsubsection{Overview of the Methods}

The framework of the relation analysis in this study is to (i) train a neural network that predicts the 3D location of each individual macaque - simulated or real-from the locations of the other individuals, and then (ii) check how much attention the network pays to each of the referent individuals when it makes the predictions. For simplicity, the network receives the location data at a single time point only and does not analyze temporal changes in the data, although the temporal information could include important traits of individuals and groups (cf. Morita et al., 2020) and thus should be discussed in future studies.

Figure 3 shows a sketch of the network structure, without the details. The location of an individual named $A$ is predicted from that of four other individuals, $B, C, D$, and $E$. The prediction is made from the weighted sum of the (transformed) location information of the four referents, where the relevance of each referent to $A$ - called attention weight - is also derived from the location information. The attention weights govern the information flow from the referents; they intuitively represent who is contributing how much to the prediction. Our analysis of the relations between the target and the referents is performed on these attention weights.

The attention weights were normalized and always have a sum of 1 . An inherent disadvantage of this formalization is that the model never detects the independence of the target individual, as at least one referent will have a non-zero weight. To get around this, the predictive performance of the attention model 


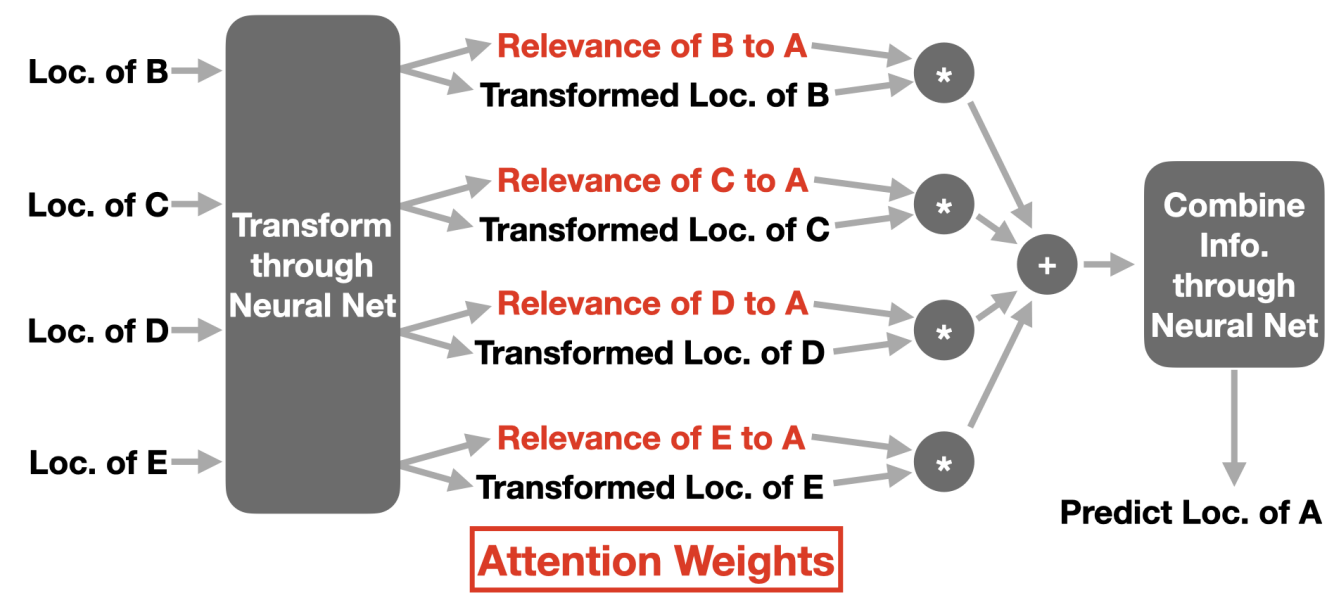

Figure 3: Schematic diagram of attention-based analysis.

was compared with a baseline model that predicts the target without reference to the other individuals. If the attention weights are redundant, the attention model will have the same level of predictive performance as the no-referent baseline. The baseline model was trained to maximize the likelihood. ${ }^{1}$

\subsubsection{Detailed Description of the Neural Network Structure}

This section provides a detailed description of the attention-based neural network used in this study. As explained in the previous section, the neural network predicted the discretized location $y_{i} \in\{1, \ldots, C\}(C=$ 400) of each individual $i \in\{1, \ldots, N\}(N=5)$ from the continuous 3D location of the other $N-1$ individuals, $\mathbf{x}_{1}, \ldots, \mathbf{x}_{i-1}, \mathbf{x}_{i+1}, \ldots, \mathbf{x}_{N}\left(\mathbf{x}_{j} \in \mathbb{R}^{3}\right)$. The predictions were made in the form of conditional probability: that is, $\mathbb{P}\left(Y_{i}=y_{i} \mid \mathbf{x}_{1}, \ldots, \mathbf{x}_{i-1}, \mathbf{x}_{i+1}, \ldots, \mathbf{x}_{N}\right)=: \pi_{i, y_{i}}$. To efficiently compute all the combinations of the target and referent individuals, the researchers customized Transformer (Vaswani et al., 2017; Devlin et al., 2018) to fit the study's purpose (i.e., the analysis of inter-individual relations), preventing the undesirable flow of information (e.g., the location of an individual should not be used when predicting that individual's own location). This allowed us to represent the input (continuous 3D location) and output (conditional probability of each possible discretized location) of all the individuals in each single matrix, which can be expressed as $\mathbf{X}:=\left(\mathbf{x}_{1}, \ldots, \mathbf{x}_{N}\right)^{\mathrm{T}}$ and $\boldsymbol{\Pi}:=\left(\pi_{i, c}\right)_{i \in\{1, \ldots, N\}, c \in\{1, \ldots, C\}}$ respectively. The $\mathbf{x}_{j}$ was scaled (by the upper bound in each dimension, see $\S 2.2$ for the bounds) and shifted such that each dimension ranged between -1 and 1 .

Figure 4 shows the network architecture, which is composed of two attention layers. Attention layers are a neural network module that take two types of inputs: query $(\mathbf{Q})$ and memory (M). In the current

\footnotetext{
${ }^{1}$ As reported in $\S 2.2$, the location of the target individual was discretized in order to improve the performance of the neural networks (van den Oord et al., 2016a,b) and simplify the maximum likelihood training of the baseline model (i.e., the occurrences of each discretized location value were counted and then the gathered data were normalized).
} 
study, $\mathbf{Q}$ and $\mathbf{M}$ were matrices of the same size whose rows represented the $N$ individuals and whose columns represented the arbitrary number of dimensions in hidden layers. The memory encoded the location information of the referent individuals. The query, on the other hand, was either a vector-represented identity information of the target individuals (the first layer) or the output from the first attention layer (the second layer). The memory was linearly transformed into two matrices of the same size, called key (K) and value ( $\mathbf{V}$; see Figure $5 \mathrm{~b})$. The core computation in the attention layers was a weighted summation of the row vectors of $\mathbf{V}$, which corresponds in this study to combining the information of the referent individuals proportional to their importance. The weights for this summation (i.e., the attention weights) were computed from the dot product of the row vectors of the key and query; equivalently, the matrix multiplication $\mathbf{K Q}^{\mathrm{T}}$ was computed. The attention weights were obtained by scaling the dot product by the square root of the hidden dimensionality (i.e., the number of the columns in the key and query) and normalizing the outcome so that the sum over the referent individuals (i.e., rows of $\mathbf{K Q}^{\mathrm{T}}$ ) was 1.0.

In NLP applications, attention layers are usually split into several computations in parallel, called heads. Those multiple heads are implemented simply by splitting the hidden dimensions of the query, key, and value after the first linear transform (i.e., each head performs the computation using their submatrices grouped by columns; see Figure 5b). ${ }^{2}$ Having multiple heads often improves model performance because different heads can have different attention weights and, thus, different roles. In machine translation, for example, some heads may concentrate their attention weights on a single word in the source language upon the prediction of the corresponding word in the target language, while other heads may distribute their attention weights more broadly to incorporate the context of the source language sentences. In the current study, the first and second attention layers had different numbers of heads owing to their different roles. On one hand, the second layer was single-headed and its attention weights were used to analyze inter-individual relations; this simpler architecture made interpretation of the attention weights easier. On the other hand, the role of the first layer was to provide useful information to the second layer (see the next paragraph for more details), and it was not used for the analysis of inter-individual relations. Thus, multiple heads were implemented in the first layer to improve model performance of the training objective.

The major difference between this study and standard applications of Transformer/self-attention is that here, the network only uses the input location data as the memory of the attention. If we also use the input location for the query, then the network would be notified which individual is geometrically closest to the target (as the attention weights are given by the similarity between the query and the key, which is computed from the memory) and may simply use that closest individual for the prediction. This is not

\footnotetext{
${ }^{2}$ The scaling of the dot product of the key and query is based on the hidden dimensionality of each head. That is, the scalar is $\sqrt{d / h}$ where $d$ is the hidden dimensionality before head-splitting and $h$ is the number of heads.
} 


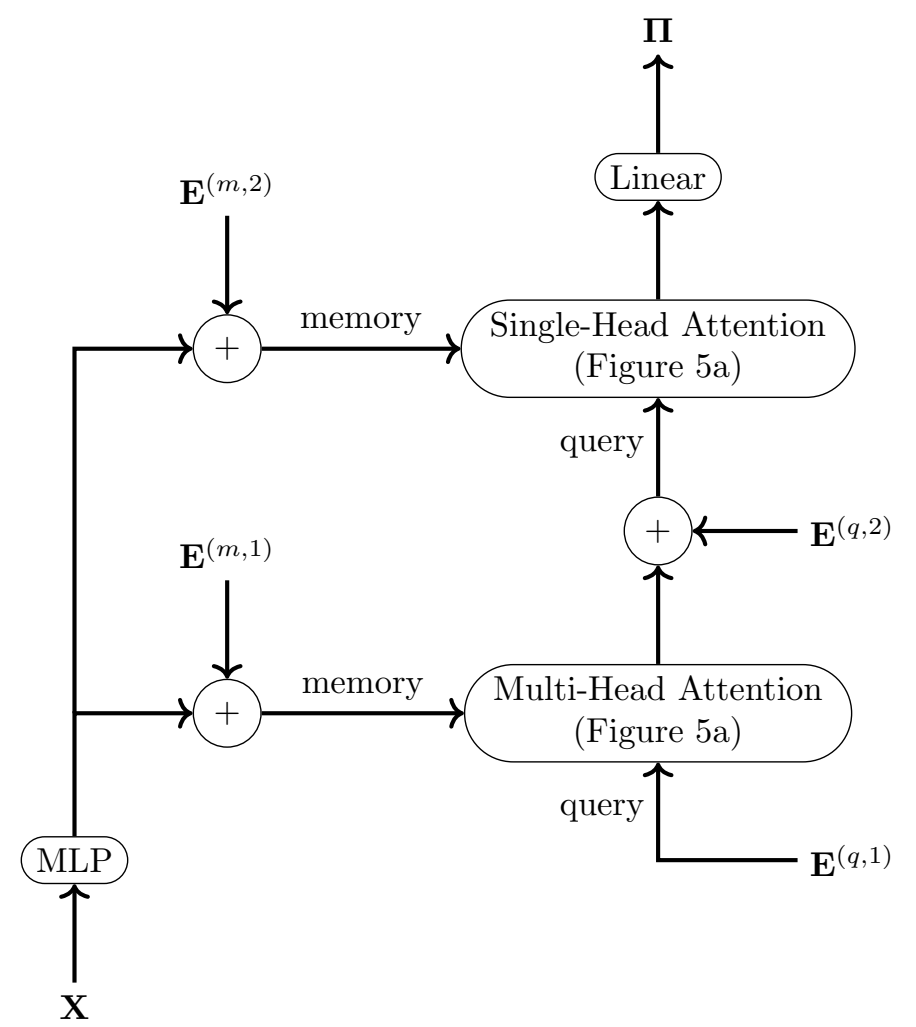

Figure 4: The entire structure of the neural network.

the intended interaction of the investigation. Instead, in the first attention layer, the query only contained information about whose location was being predicted as the target, which was represented by trainable individual embeddings $\mathbf{E}^{(q, 1)}$ (cf. akin to the time encoding in Shaw et al., 2018; Dai et al., 2019). The second layer received the output from the first layer-in which the location of each referent individuals (excluding the target) was encoded in a single vector per target-and used it as its query, together with other individual embeddings $\mathbf{E}^{(q, 2)}$. The second layer therefore computed the attention weight of each referent individual from three sets of information: (i) the individual's location (memory), (ii) the location of the other referents (query, output from the first layer), and (iii) the name of the target individual being predicted (query), while the first layer had access only to (i) and (iii).

Note that the input location vector $\mathbf{x}_{i}$ and its transformed representation after the multilayer perceptron (MLP) did not encode who was located in that location (i.e., the network did not see the " $i$ ", the row index in the matrix $\mathbf{X}$; cf., the necessity of time-encoding in Transformer). This is why we also added the individual embeddings $\mathbf{E}^{(m, 1)}$ and $\mathbf{E}^{(m, 2)}$ to the memory.

Other customizations inside the two attention layers are highlighted in yellow in Figure 5. First, we masked the diagonal entries of the attention weight matrix (Figure 5b); otherwise, the network allows redundant predictions whereby a target individual's location could be identified from its own input value. 


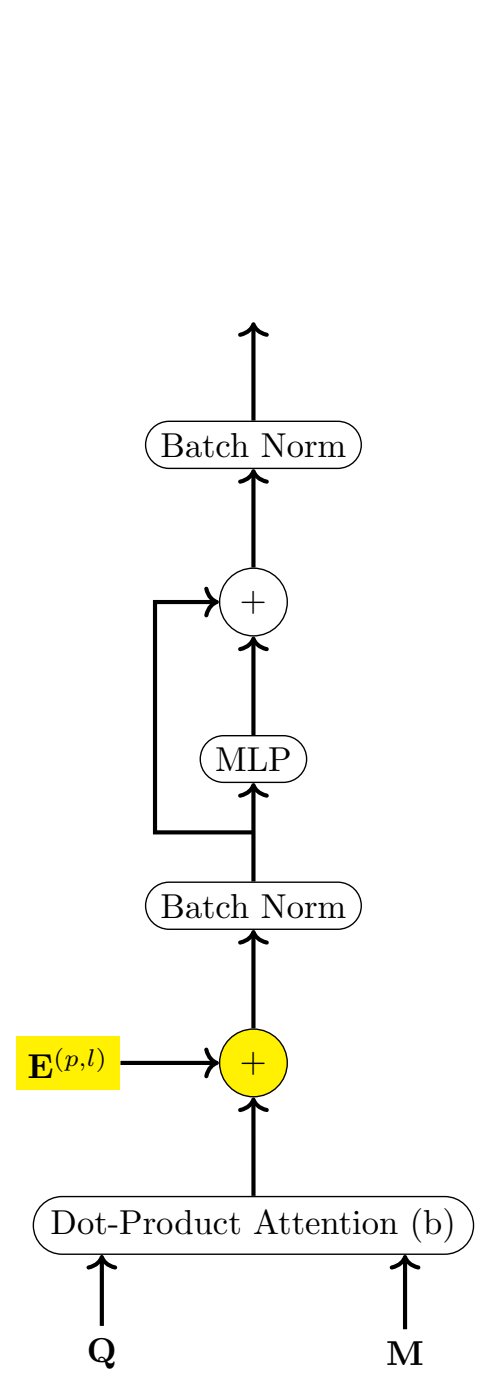

(a) Attention Layer

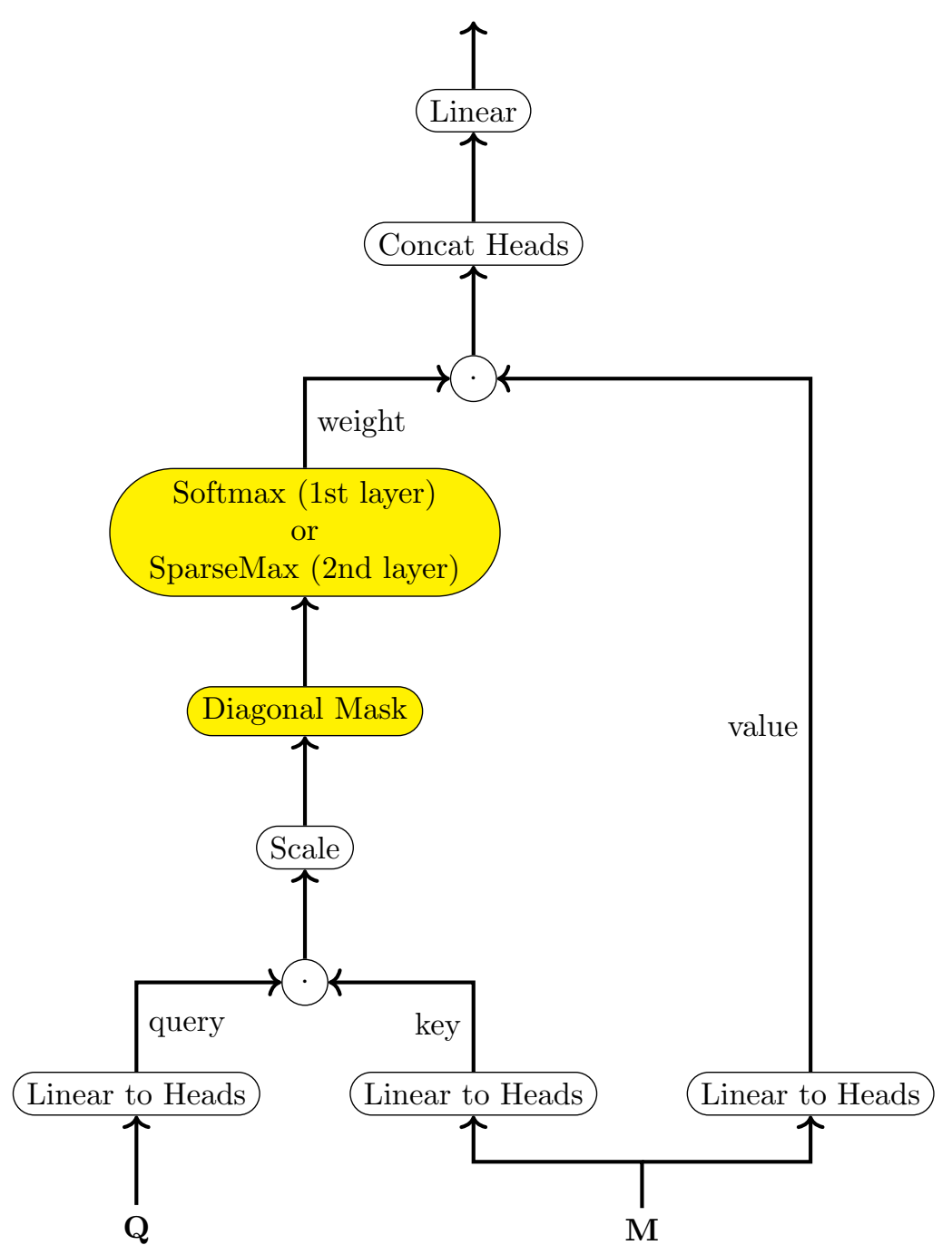

(b) Single/Multi-Head Dot-Product Attention

Figure 5: Structure of the attention layers (a) and their single/multi-head dot-product attention module (b). The nodes highlighted in yellow include modifications to the canonical design in Transformer. 
Secondly, the softmax was replaced with the sparsemax (Martins and Astudillo, 2016; Niculae and Blondel, 2017; Niculae et al., 2018) to compute the attention weights in the second layer (Figure 5b), where the analysis of inter-individual relations was performed. This change made the analysis more robust and interpretable because the sparsemax can assign zeros to unmasked entries and express "no contribution", whereas the softmax always assigns non-zero weights. The softmax was kept in the first layer to create a single-vector representation of all the referent individuals, and the non-zero weights assigned by the softmax were found to be appropriate. Finally, the residual connection around the dot-product attention was removed (Figure 5a; the other residual connection around MLP was kept) because, aside from the information gated by the attention module, the residual connection also leaks information about the referent location, which makes the attention weights uninterpretable. Instead of the residual connection, individual embeddings $\mathbf{E}^{(p, l)}$ (where $l \in\{1,2\}$ denotes the layer) were added to the output from the dot-product attention. Since we masked the diagonal entries of the attention weight matrix, the output lost the information about who was being predicted. Hence, we needed to retrieve that information before the final predictions were made based on the output from the second attention layer. ${ }^{3}$

All the hidden units in the network had 512 dimensions, except that the dimensions were evenly split among the four heads (128 dimensions each) in the attention layer.

\subsubsection{Training Procedure}

The network was trained for 30,000 iterations. The researchers used the Adam optimizer with a learning rate of $0.005, \beta_{1}=0.9, \beta_{2}=0.999$, and a weight decay of 0.01 . The learning rate was updated based on Transformer studies (Vaswani et al., 2017; Devlin et al., 2018), with 3,000 warm-up iterations. The batch size was 512. Dropout was applied at a rate of 0.1 to the output from the MLPs (Figure 4,5a) and the dot-product attention (Figure 5a before the addition of $\mathbf{E}^{(p, l)}$ ), but was not applied to the attention weights. This is because the number of attendees was small (five) in this study and robust inference is needed for that part.

\section{$2.2 \quad$ Data}

Attention-based relation analysis was performed on four simulated data (§2.2.1) and one real data (§2.2.2).

\footnotetext{
${ }^{3}$ Note that $\mathbf{E}^{(p, 1)}$, the post-attention embeddings in the first attention layer, duplicate the role of $\mathbf{E}^{(q, 2)}$, which constitutes the query input of the second layer. One of them can therefore be removed, in principle.
} 


\subsubsection{Simulated Data}

The reliability of the attention-based relation analysis was tested on four types of simulated data. Following the real data collected from Japanese macaques (see $§ 2.2 .2$ ), both simulations had five individuals located in a bounded $3 \mathrm{D}$ space $([0.0,5.0] \times[0.0,4.0] \times[0.0,2.5])$, resembling the group cage where the macaques were kept.

The four simulations consisted of two binary factors. The first factor governed relational structures of the animal agents. One of the structures modeled the scenario in which the five agents moved in a sequence, each following the other (Figure 6a). The other structure modeled the hub and spoke relation in which a single hub individual was followed by the other four spoke dependents (Figure 6b).

The second factor parameterized pairwise dependency patterns between the related agents. One of the dependence patterns modeled a proximal situation in which each follower agent was always positioned around its precedent agent. Specifically, the serial-follower condition located the $(i+1)$-th individual uniformly at random in the intersection of the domain and the sphere with a radius of 1.5 centered at the $i$-th individual's location, which was implemented by rejection sampling (Figure 6c). Similarly, the hub-spoke condition sampled the location of the spoke individuals uniformly from the intersection of the domain and the sphere with a radius of 1.5 centered at the hub individual's location (Figure 6d). By contrast, the other dependence pattern modeled a less close relation in which the follower kept a certain distance from the precedent. In the serial-follower condition, the location of the $(i+1)$-th individual was sampled uniformly at random from the surface of the sphere with a radius of 1.5 centered at the $i$-th individual's location, ensuring that the sampled location was bounded in the domain (by rejection sampling; Figure 6e). Likewise, the spoke individuals in the hub-spoke condition were located uniformly at random on the surface of the sphere with a radius of 1.5 centered at the hub individual (Figure 6f). In all the four simulations, the non-follower's location (i.e., the first individual in the serial condition and the hub individual in the hub-spoke condition) was sampled uniformly randomly from the domain.

For each of the four simulations, 100,000 samples were generated-each containing 3D coordinates of the five individuals. ${ }^{4}$ All the samples were used for training and attention analysis. The researchers did not hold out a test portion of the data, as the study's purpose was not to test the general applicability of the model predictions, but rather to reveal the dependency relations in the data (just as in linear regression tests).

Note that in all the four cases, the location samples were the only information available to the neural network analyzer. Furthermore, the settings of the simulations, including the types of inter-individual relations and the form of the conditional distribution, were not hard-coded in the analyzer, unlike with other

\footnotetext{
${ }^{4}$ The number of different target-referents pairs observed by the neural network was $5 \times 100,000=500,000$, since all the possible partitions of the five individuals were treated in the analysis as one target and four referents.
} 


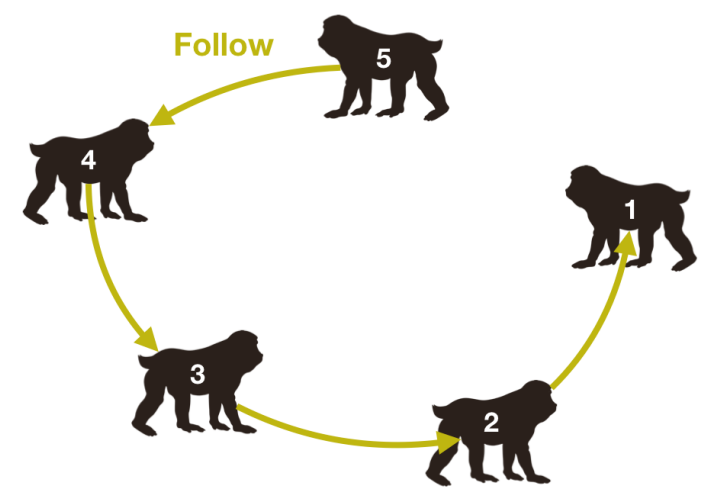

(a) Serial Followers

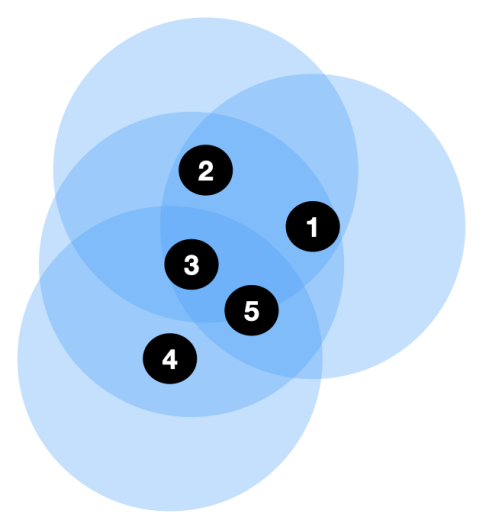

(c) Proximal Serial Followers

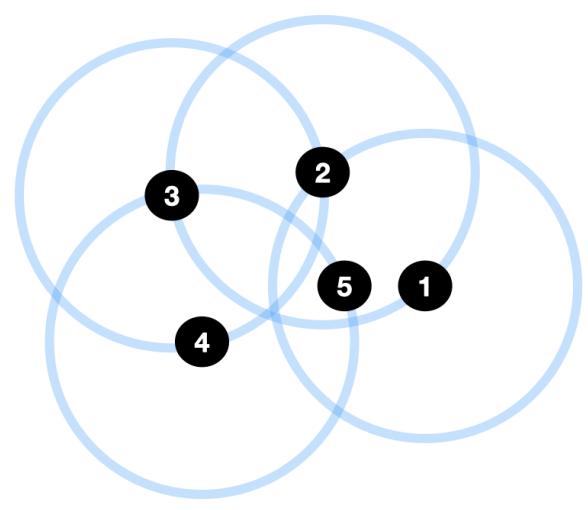

(e) Distant Serial Followers

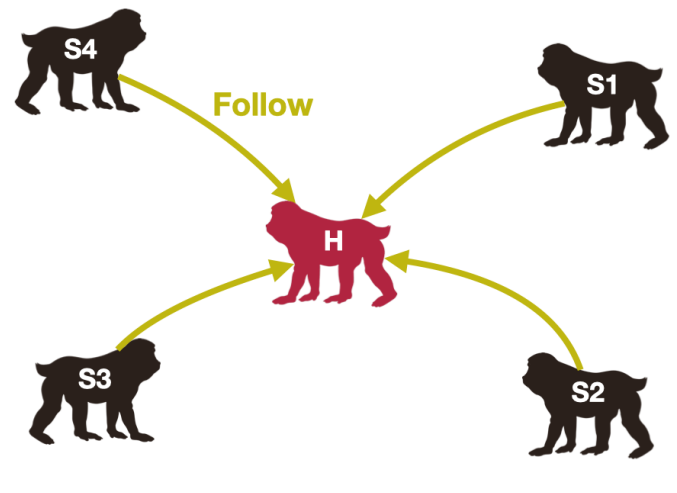

(b) Spoke Followers

(d) Proximal Spoke Followers

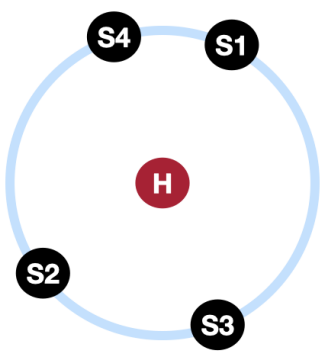

(f) Distant Spoke Followers

Figure 6: Two patterns of simulated data. (a) and (c) model animal agents that move in a sequence. (b) and (d) model the situation where there is a single hub individual (red) that is followed by the other four spoke dependents (black). (a) and (b) depict the relations among the animal agents modeled in the simulations. (c) and (d) describe the distributions from which the location data are sampled: The blue circles represent the support of the uniform distribution centered at each following individual. 
classical analyses assuming a limited hypothesis space including the correct generative model (e.g., Bayesian inference). For example, when analyzing the data from the hub-relation simulations, the network had to (i) discover the hub structure among many other possible relation patterns behind the data, and (ii) identify the hub individual while the five agents were always close to one another, without being tricked by different distributional patterns (proximal or distant).

Although the simulated data took on continuous values (residing in the bounded subspace of the $3 \mathrm{D}$ Euclidean), it has been reported that neural networks are often better at predictions of discrete categories (due to the flexibility of categorical distributions; van den Oord et al., 2016a,b, similar discrete predictions of location data were also adopted by Takeda and Komatani, 2016). Accordingly, the domain was split into 400 cubes $(0.5 \times 0.5 \times 0.5)$, and the network was trained to make a discrete prediction of the cube containing the target's location.

\subsubsection{Real Data}

In addition to the simulated data described in $\S 2.2 .1$, we analyzed real 3D location data of five captive Japanese macaques in the Primate Research Institute, Kyoto University (KUPRI), Japan. The subjects were two adult males and three adult females. Location recordings of the five macaques were conducted for two weeks in an outdoor group cage $(4 \times 5 \times 2.5 \mathrm{~m})$ where the subjects were allowed to freely move and interact with one another. Each subject carried two Bluetooth ${ }^{\circledR}$ Low-Energy beacons, and 3D location was estimated based on the signals coming from these beacons (Quuppa Intelligent Locating System ${ }^{\mathrm{TM}}$ ). See Morita et al. (2020) for more details on the data collection procedures.

Although the system sampling frequency was set at $9 \mathrm{~Hz}$, the actual rate was unstable because of uncontrollable signal interference and reflection perturbation. The sampling from the $2 \times 5=10$ beacons did not also synchronize their timing. We therefore took the median value of the samples from each individual (i.e., from the two beacons) for each dimension collected at every 3000 msec interval. The medians over the same interval constituted the input-output pair for the neural network. ${ }^{5}$ We note that taking medians decreased time resolution of the collected data; however, temporal information was ignored in the analysis. The preprocessing above yielded a total of 327,592 sets of data. ${ }^{6}$ Just as in the simulations, all the data was used for both the model training and attention analysis without holding out a test portion, and the target locations were discretized into 400 cubes $(0.5 \times 0.5 \times 0.5 \mathrm{~m})$.

\footnotetext{
${ }^{5}$ The network simultaneously analyzed all possible choices of one individual as the prediction target and of four individuals as the referents.

${ }^{6}$ Again, the researchers analyzed all possible partitions of the five individuals into one target and four referents; the number of different target-referent pairs observed by the neural network was therefore $5 \times 327,592=1,637,960$.
} 


\section{Results}

The attention-based analysis correctly distinguished the two relational structures in the simulations (serial and hub-spoke relations) regardless of the distance pattern between the agents (proximal or distant). Given the data from the serial follower simulation, the analyzer correctly assigned heavy weights $(>0.2)$ to the relations between the individuals with successive indices (e.g., "2" and "3"; Figure 7a,c, 8a,c). On another note, the hub-spoke simulation concentrated the attention weights to the hub individual when predicting the spoke dependents, and evenly distributed the weights to the spokes when predicting the hub (Figure $7 \mathrm{~b}, \mathrm{~d}$, $8 \mathrm{~b}, \mathrm{~d})$. Note that the analysis of the four different simulations was performed using identical free parameters and was therefore non-parametric.

Unlike our proposed method, traditional distance-based analyses cannot capture the correct relational patterns. Specifically, the relations among the distant followers cannot be captured from their Euclidean distance, which was by definition constant between related individuals (at 1.5), while non-related individuals became closer for about $50 \%$ of the time (Figure 8e,f). Hence, the non-related individuals would be incorrectly identified as related if, for example, the approaches under 1.5 were counted. Distance-based analyses would also be less robust for the proximal patterns. While the related individuals were at most 1.5 apart (by definition), the non-related individuals were located closer than 1.5 for $39-63 \%$ of the time (Figure 7 e,7f). Thus, it is hard to achieve a robust contrast between the related and non-related individuals from the distance information, especially to the extent achieved by the attention-based analysis (Figure $7 \mathrm{a}-\mathrm{d}$ ).

It should be noted that the detected relations are asymmetric but do not correspond to the causal structures in the simulations. Instead, the model computes the conditional probability of the location of each target individual given that of the referent individuals, and the attention weights represent the contribution of each referent individual to the computation of the conditional probability. For example, the locations of the individual indexed " 1 " in the serial-follower simulations and the hub individual in the spoke-follower simulations were sampled unconditionally from the domain, without reference to any other individuals. However, the model can locate these individuals more accurately if it refers to their followers (the second and spoke individuals, respectively); in other words, the conditional probability, $\mathbb{P}\left(y_{1 / \mathrm{H}} \mid \mathbf{x}_{-1 / \mathrm{H}}\right)$ (where $y_{1 / \mathrm{H}}$, is the (discretized) location of the leading/hub individual and $\mathbf{x}_{-1 / \mathrm{H}}$ is the (continuous-valued) location of the other individuals), rewritten as follows by Bayes' Theorem:

$$
\mathbb{P}\left(y_{1 / \mathrm{H}} \mid \mathbf{x}_{-1 / \mathrm{H}}\right)=\frac{\mathbb{P}\left(y_{1 / \mathrm{H}}\right) \mathbb{P}\left(\mathbf{x}_{-1 / \mathrm{H}} \mid y_{1 / \mathrm{H}}\right)}{\mathbb{P}\left(\mathbf{x}_{-1 / \mathrm{H}}\right)} \neq \mathbb{P}\left(y_{1 / \mathrm{H}}\right)
$$




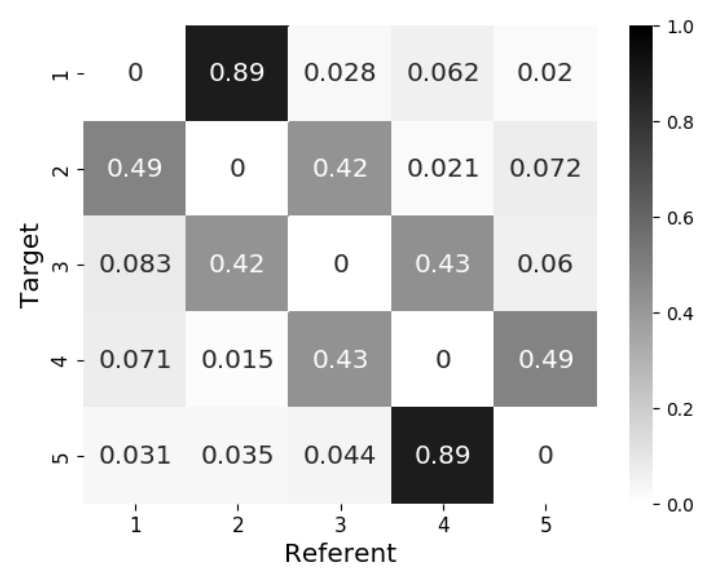

(a) Attention Weights (Proximal Serial Followers)

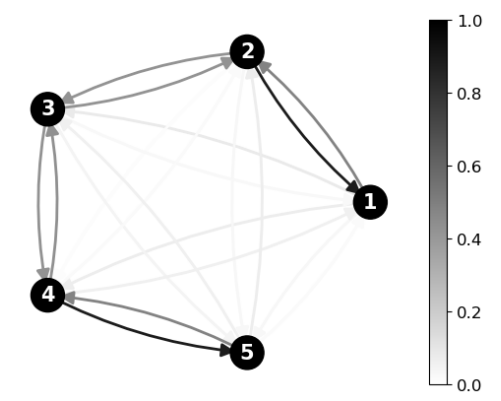

(c) Attention Weights (Proximal Serial Followers)

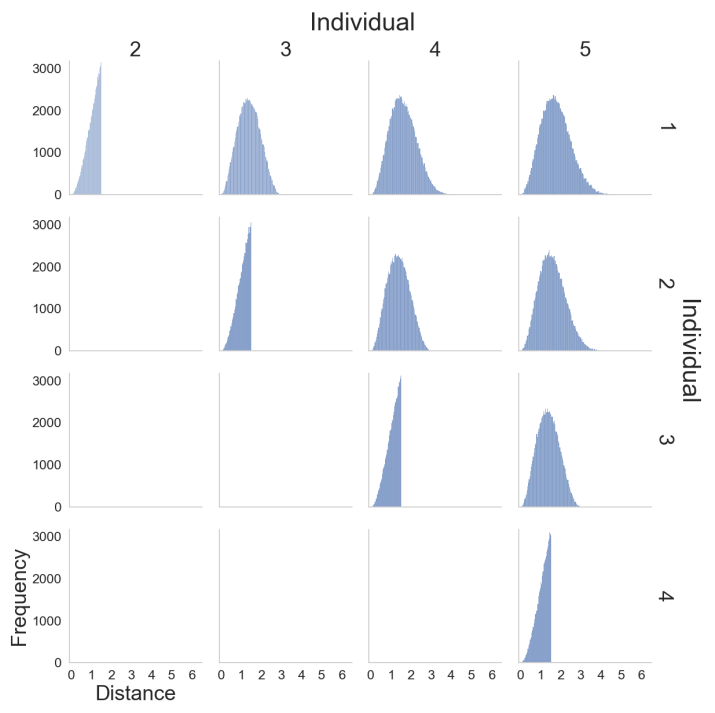

(e) Pairwise Distance (Proximal Serial Followers)

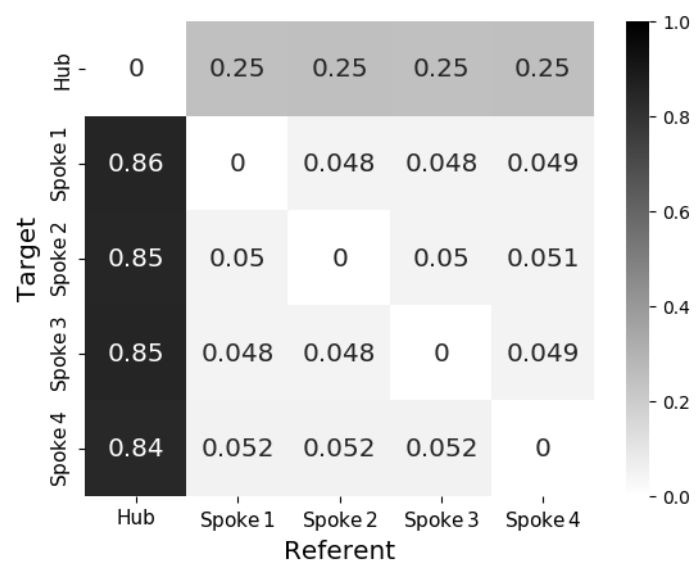

(b) Attention Weights (Proximal Spoke Followers)

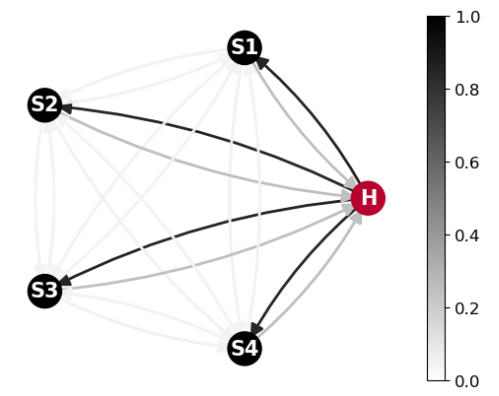

(d) Attention Weights (Proximal Spoke Followers)

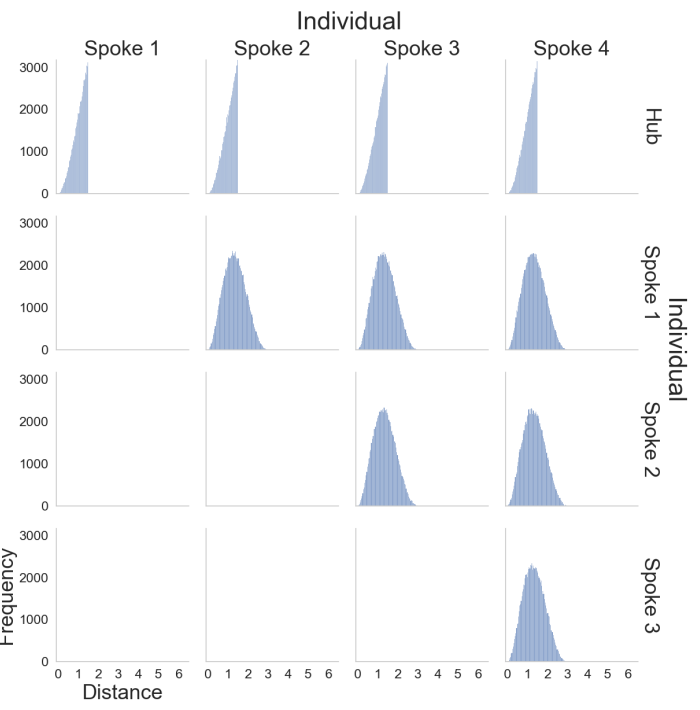

(f) Pairwise Distance (Proximal Spoke Followers)

Figure 7: Analysis of proximal-follower simulations (left column: serial followers, Figure 6c; right column: spoke followers, Figure 6d). Top (a,b): Heatmap representation of average attention weights assigned to referent individuals (columns) upon the prediction of the location of target individuals (rows). Middle (c,d): Graphical representation of average attention weights assigned to referent individuals upon the prediction of the location of target individuals (i.e., the same data as the top, presented in a different format). Each arc runs from a referent to a target individual, with attention weight represented by color density. Bottom $(\mathrm{e}, \mathrm{f})$ : Distribution of the pairwise 3D Euclidean distance between the individuals. 


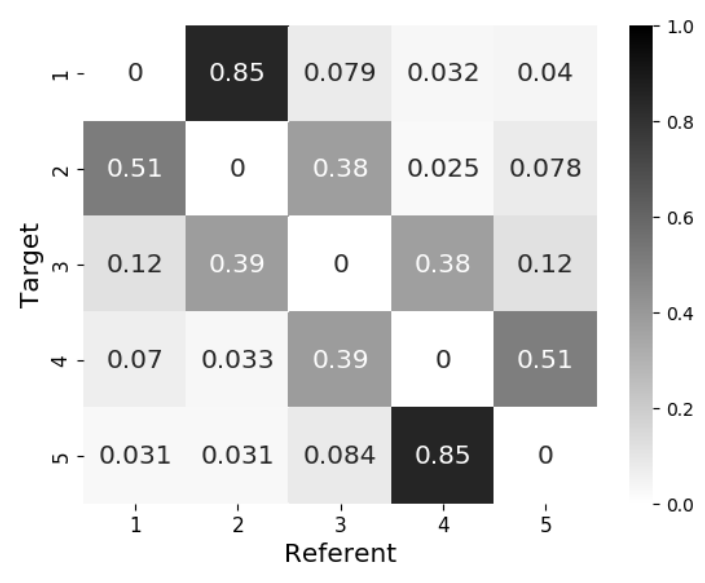

(a) Attention Weights (Distant Serial Followers)

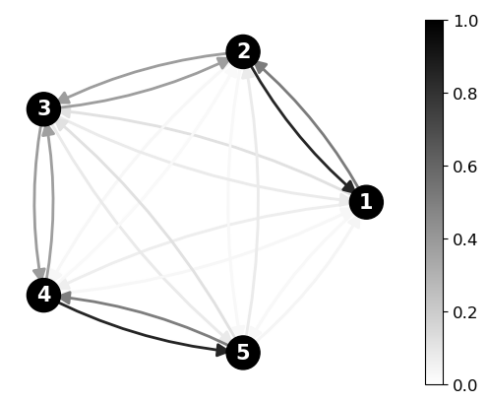

(c) Attention Weights (Distant Serial Followers)

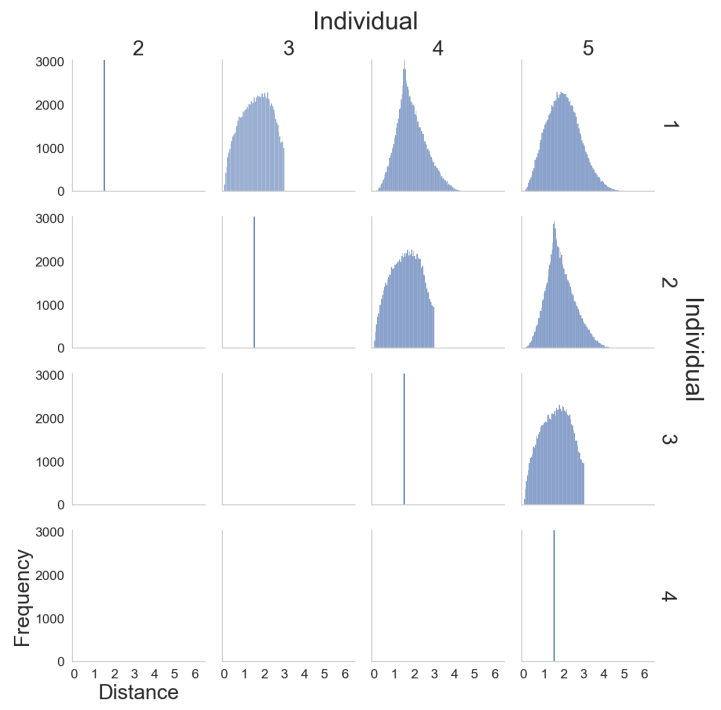

(e) Pairwise Distance (Distant Serial Followers)

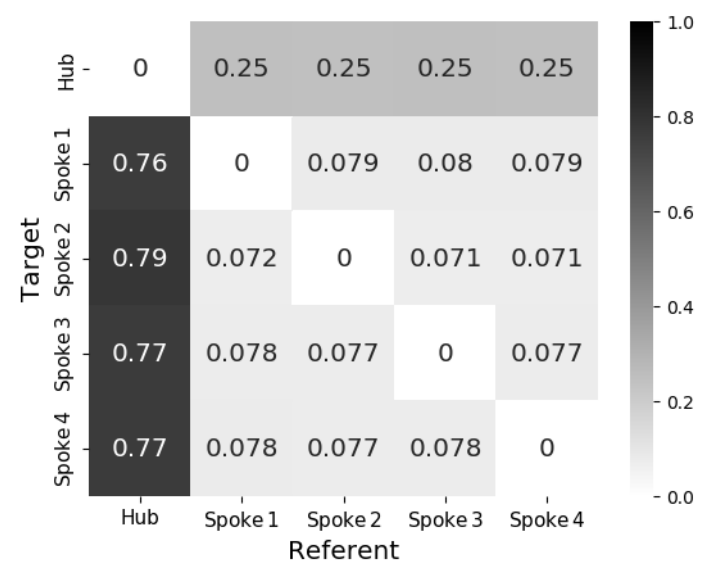

(b) Attention Weights (Distant Spoke Followers)

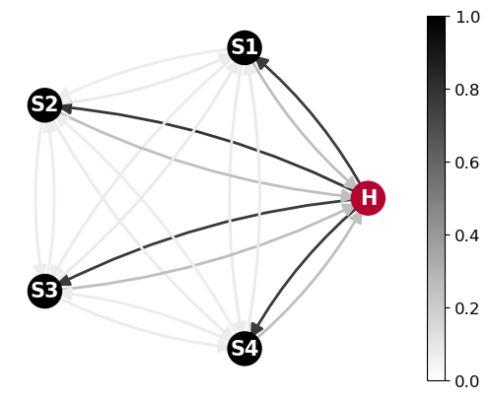

(d) Attention Weights (Distant Spoke Followers)

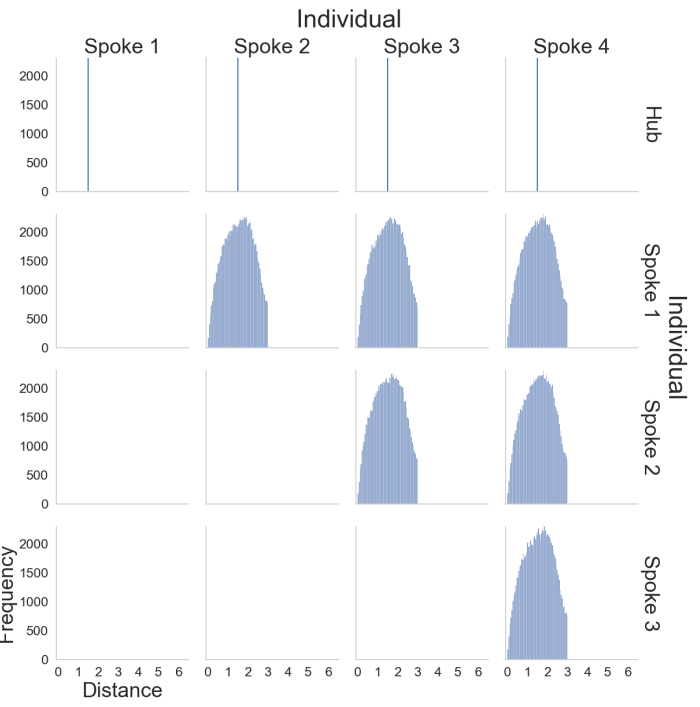

(f) Pairwise Distance (Distant Spoke Followers)

Figure 8: Analysis of distant-follower simulations (left column: serial followers, Figure 6e; right column: spoke followers, Figure 6f). Top (a,b): Heatmap representation of average attention weights assigned to referent individuals (columns) upon the prediction of the location of target individuals (rows). Middle (c,d): Graphical representation of average attention weights assigned to referent individuals upon the prediction of the location of target individuals (i.e., the same data as the top, presented in a different format). Each arc runs from a referent to a target individual, with attention weight represented by color density. Bottom $(\mathrm{e}, \mathrm{f})$ : Distribution of the pairwise 3D Euclidean distance between the individuals. 
because $\mathbb{P}\left(\mathbf{x}_{-1 / \mathrm{H}} \mid y_{1 / \mathrm{H}}\right) \neq \mathbb{P}\left(\mathbf{x}_{-1 / \mathrm{H}}\right)$ (i.e., the follower individuals are dependent on the leading/hub individual). Thus, non-zero attention weights should be assigned to the follower individuals.

While it was not capable of detecting the original causal relations, the proposed method distinguished direct vs. indirect statistical dependencies in the simulations. For example, the individuals indexed " 1 " and " 3 " are only conditionally independent given the location of the individual indexed " 2 "; that is, if the second individual is not taken into account, the first and third individuals are located more accurately by reference to the location of each other and thus are statistically dependent. This implies that naive pairwise evaluation of statistical dependence would fail to achieve the global structure consisting of direct dependencies. By contrast, the attention-based analysis successfully detected only the direct dependencies among a number of possible relational structures.

The attention-based analysis of the real data revealed that the female macaques were more informative referents than the males, especially for predicting the other females. The attention weight to Male 1 as a referent was smaller than those of all the females, except when Male 2 was predicted (where Female 1 collected zero attention). ${ }^{7}$ Further, and Male 2 was almost never used as a referent (Figure 9a,b). It is of note, however, that the males did not move independently of the other individuals. The predictions of the males' location were based on three other individuals (Male 1 was dependent on the females, and Male 2 was dependent on Female 2 and 3 and Male 1). While the neural network was unable to flag independence, as the attention weights were never all zeros, these detected dependencies were not redundant because the network outperformed the no-referent baseline. As shown in Figure 9c, the average prediction loss (i.e., negative log probability) of the attention model was smaller than the baseline across all the individuals.

As with the simulation data, the real data challenge traditional distance-based analyses. The Euclidean distance between the macaques showed three frequency peaks: approximately 0.0-1.5, 1.5-3.0, and 3.0-4.5 m (Figure 9d). These multimodal distributions can originate either from social relations among the macaques or from other factors: for example, a small climbing frame and a hammock were installed around the center and peripherals of the experimental cage (see Figure S1 in the supporting information) and the second peak of the distance distribution could be caused by monkeys sitting on those facilities. In the current case, the canonical proximity analysis - focusing only on the leftmost peaks - seems to agree with the attention-based analysis; for example, both the analyses suggest that Male 2 (taking greater distances from the others and receiving almost zero attentions as a referent) was isolated from the other macaques. Note, however, that the ignorance of the second and third peaks in the distributions was non-trivial and unjustified unless the more non-parametric analysis was performed.

\footnotetext{
${ }^{7}$ The average attention to Male 1, upon the predictions of Male 2, appeared equal to the attention to Female 3, but was statistically significantly smaller given the 10,000 samples of bootstrapping.
} 


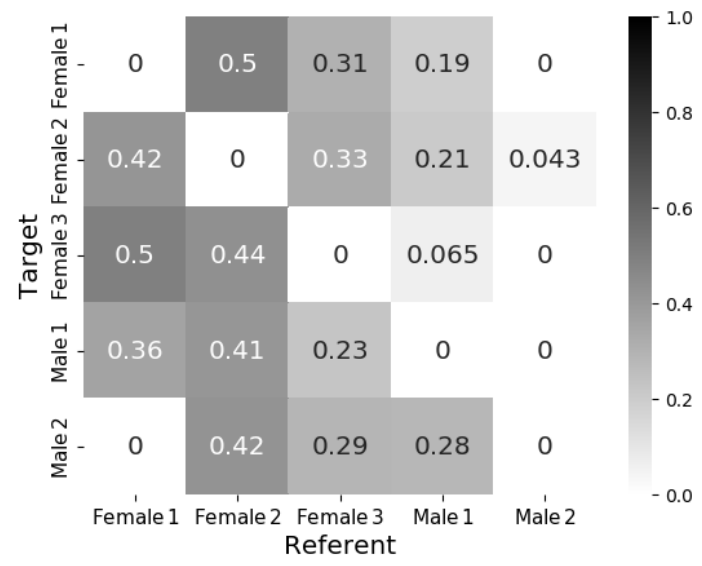

(a) Attention Weights (Real Data)

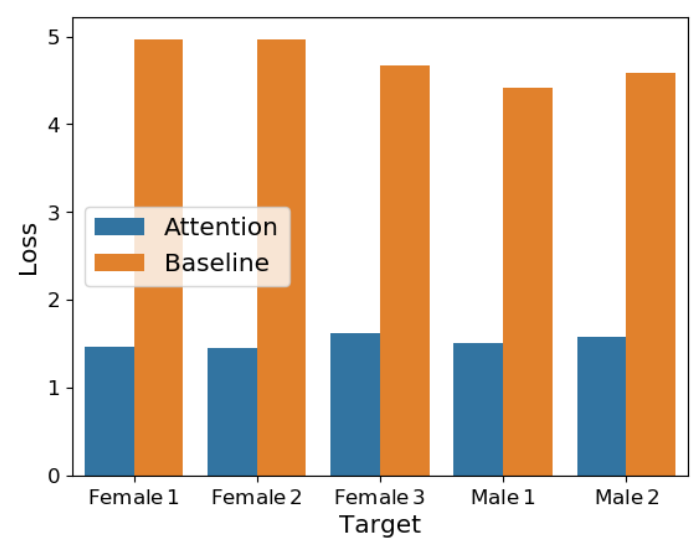

(c) Average Prediction Loss (Real Data)

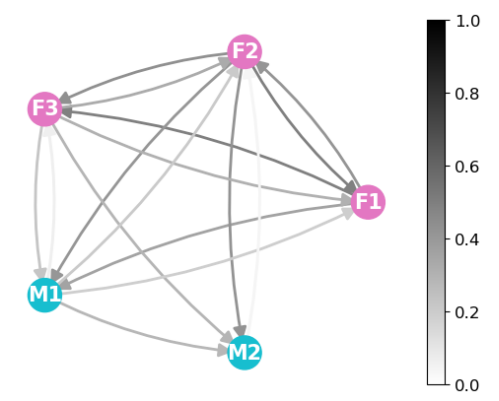

(b) Attention Weights (Real Data)

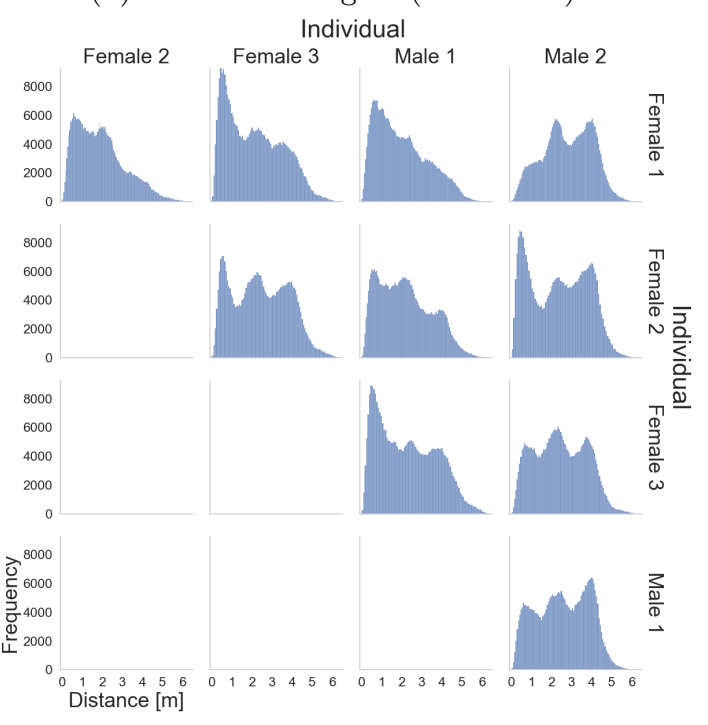

(d) Pairwise Distance (Real Data)

Figure 9: Analysis of real data collected from Japanese macaques. (a) Heatmap representation of average attention weights assigned to referent individuals (columns) upon the prediction of the location of target individuals (rows). (b) Graphical representation of average attention weights assigned to referent individuals upon the prediction of the location of target individuals (i.e., the same data as a, presented in a different format). Each arc runs from a referent to a target individual, with attention weight represented by color density. (c) Average prediction loss (negative log probability) of the attention-based neural network (blue) and the no-referent baseline (orange). (d) Distribution of the pairwise 3D Euclidean distance between the individuals. 


\section{Discussion}

In the study, the attention analysis correctly identified the two latent relational patterns behind the simulation, one having serial followers and the other having the spoke followers. While these simulations were simple, it is of note that the analyses were performed without prior assumptions about the possible types of inter-individual relations or random distributions involved. Compared with traditional distance/closenessbased analysis (Silk et al., 2006b,a; Croft et al., 2008; Clark, 2011; Boyland et al., 2013; Castles et al., 2014; Krause et al., 2015; Schofield et al., 2019; Gelardi et al., 2020), this method is likely more effective in visualizing latent dependency patterns, especially when little is yet known about the properties of data. Specifically, analysis of proximal co-appearance cannot identify related individuals when they keep their distance from one another, while the attention-based analysis successfully detected the latent relations using exactly the same values on the free parameters as for the proximal pairwise relations.

This study also demonstrated that the attention analysis is applicable to raw real data, in this instance a case study of Japanese macaques. We found that the location of females is more informative than that of males for locating the other female individuals. This result is consistent with the female-oriented social system of the macaques: Japanese macaques are a female-philopatric species (represented by parturition in the natal group and male emigration), and females usually build firmer and more stable social relationships with one another than do the males (e.g., matrilineal social rank succession; Yamada, 1963; Furuichi, 1984; Mitani, 1986; Nakagawa et al., 2010). The females of the species are also reported to exhibit more tight and frequent spatial cohesion (Otani et al., 2014), so knowing the location of some females will increase the ability to locate other females in the same group. In addition, our earlier research on the same data showed that the males' movements (i.e., trajectories of the 3D location) reflect a greater degree of individuality than that of the females (Morita et al., 2020). Because of such idiosyncratic movements, the males' locations may not be informative predictors of other individuals' locations. The analysis of the real data also revealed that one individual (Male 2) was almost never attended by the others. The discovery of such distinguished individuals is useful to build new hypotheses about animal societies and plan behavioral experiments targeting them. The attention-based analysis detects (non-)related individuals without presupposing specific types of relations, so the detected relations may reveal hitherto unknown patterns of social structures not necessarily based on proximal relations (Figure 1). Identifying and targeting (non-)related individuals may also allow for more efficient experiments/observations, especially when a large-scale animal group is studied and investigation of every single pair is impractical.

Attention-based neural networks are now being adopted in biological studies. Yuan and Jia (2019) used the attention mechanism to implement a seizure detector based on electroencephalogram (EEG) data. They 
showed that the attention mechanism can locate EEG channels that specifically diagnose seizures. Similarly, Maekawa et al. (2020) analyzed movements of worms and mice using the attention mechanism, and highlighted their characteristic movements under different experimental conditions. These studies reemphasize that attention-based data analysis targets situations in which researchers do not know which aspects/features/portions of the data are important and therefore need data-driven detection of these features. Although such non-parametric analyses have also been attempted using information-theoretic methods, those approaches often have limitations compared to deep learning. For example, Chen et al. (2019, 2020) inferred causal relationship among pigeons from GPS data based on causation entropy (i.e., mutual information between an individual at discrete time $t$ and another at time $t+1$, conditioned on some other individuals at $t$ Sun et al., 2015). While the information theoretic analysis is non-parametric in principle, empirical computation includes estimation of probability distributions and therefore can spoil the non-parametricity. Chen et al. (2019) defined probability distributions based on a 2D Euclidean proximity metric, which would miss distant dependencies similar to the simulations in this study. (Chen et al., 2020) instead used 2D velocity directions that were discretized into eight regions evenly divided in the polar coordinate (i.e., $0-45^{\circ}, 45^{-}-90^{\circ}$, $\ldots$, and $\left.315-360^{\circ}\right)$. This discretization was sparse compared with that in the present study (8 vs. 400 levels) because fine-grained discretization, specifically of the conditioning variables representing the referent individuals, makes traditional statistical estimation difficult. By contrast, the attention-based analysis, based on neural networks, can exploit continuous-valued data for the conditioning variables (raw data or embeddings of discrete data), and the sparse discretization is unnecessary. Therefore, the attention-based analysis can be more detailed and non-parametric than traditional information-theoretic approaches.

Attention analysis does not exploit any behavioral patterns that are particular to Japanese macaques, and researchers can use it for location data of other animals. The analysis is particularly appropriate when the use of conventional distance/closeness metrics is unsupported; geometric proximity can be a reasonable proxy for observations of some social behaviors such as grooming (Gelardi et al., 2020), but species without such social behaviors may exhibit relatedness at a distance. We further emphasize that attention analysis is not limited to location data; the flexibility of the neural network allows its application to a wide variety of data with interacting components. For example, vocal communication has been of major interest to behavioral biologists as well as evolutionary linguists (Levinson, 2016). Attention analysis could potentially reveal latent communication flow when applied to vocalization recordings of multiple individuals. It is to be noted, however, that the proposed method needs to be extended and refined for such broader applications and for more detailed analyses of location/movement. For example, the current version does not process temporal changes in location, whereas temporal analysis is a crucial part of analyzing dynamic behaviors and vocal communication. Since the attention mechanism was developed for NLP, however, processing time 
series data should not be difficult (see Vaswani et al., 2017; Shaw et al., 2018; Dai et al., 2019, for popular ways to encode time information).

\section{Acknowledgements}

We appreciate the animal care by our technicians and research assistants as well as their suggestions and support for this project. In particular, we wish to express special thanks to Norihiko Maeda, Mayumi Morimoto, and Takayoshi Natsume for the animal arrangements, and Panasonic Solution Technologies Co. Ltd. for technical support with the equipment. We also acknowledge the very useful comments and suggestions on the analysis from Zin Arai and Hiroshi Takeuchi. This study was performed under the Cooperative Research Program at KUPRI (2018-C-27, 2019-B-27). This study was mainly funded by the Japan Science and Technology Agency, Core Research for Evolutional Science and Technology 17941861 (\#JPMJCR17A4) and partly by MEXT Grant-in-aid for Scientific Research on Innovative Areas \#4903 (Evolinguistic), 17 H06380.

\section{Ethical Statement}

All the procedures were reviewed and approved by the Animal Welfare and Care Committee of KUPRI (Permission \# 2018-203) and complied with the institutional guidelines (Primate Research Institute, 2010).

\section{Author contributions}

Project organization: IM HK; Animal arrangements: HK SA NSH; Apparatus building: AT IM HK; Data acquisition: AT IM HK; Animal cares: AK AT IM HK; Data management; HK AT TM; Computational modeling: TM; Manuscript writing: IM HK TM.

\section{Data Availability}

The data and code used in this study will become available in Mendelay Data and GitHub respectively when it is accepted for publication. 


\section{References}

Bahdanau, D., Cho, K., and Bengio, Y. (2015). Neural machine translation by jointly learning to align and translate. In 3rd International Conference on Learning Representations, ICLR 2015, San Diego, CA, USA, May 7-9, 2015, Conference Track Proceedings.

Boyland, N. K., James, R., Mlynski, D. T., Madden, J. R., and Croft, D. P. (2013). Spatial proximity loggers for recording animal social networks: consequences of inter-logger variation in performance. Behavioral Ecology and Sociobiology, 67(11):1877-1890.

Canteloup, C., Puga-Gonzalez, I., Sueur, C., and van de Waal, E. (2020). The effects of data collection and observation methods on uncertainty of social networks in wild primates. American Journal of Primatology, 82(7):e23137.

Castles, M., Heinsohn, R., Marshall, H. H., Lee, A. E., Cowlishaw, G., and Carter, A. J. (2014). Social networks created with different techniques are not comparable. Animal Behaviour, 96:59-67.

Chen, D., Kang, M., and Yu, W. (2020). Probabilistic causal inference for coordinated movement of pigeon flocks. EPL (Europhysics Letters), 130(2):28004.

Chen, D., Wang, Y., Wu, G., Kang, M., Sun, Y., and Yu, W. (2019). Inferring causal relationship in coordinated flight of pigeon flocks. Chaos: An Interdisciplinary Journal of Nonlinear Science, 29(11):113118.

Chepko-Sade, B., Reitz, K. P., and Sade, D. S. (1989). Sociometrics of macaca mulatta iv: Network analysis of social structure of a pre-fission group. Social Networks, 11(3):293-314. Special Issue on Non-Human Primate Networks.

Clark, F. E. (2011). Space to choose: network analysis of social preferences in a captive chimpanzee community, and implications for management. American Journal of Primatology, 73(8):748-757.

Croft, D. P., James, R., and Krause, J. (2008). Exploring Animal Social Networks. Princeton University Press.

Cybenko, G. (1989). Approximation by superpositions of a sigmoidal function. Mathematics of Control, Signals and Systems, 2(4):303-314.

Dai, Z., Yang, Z., Yang, Y., Carbonell, J., Le, Q. V., and Salakhutdinov, R. (2019). Transformer-XL: Attentive language models beyond a fixed-length context. 
Devlin, J., Chang, M.-W., Lee, K., and Toutanova, K. (2018). BERT: Pre-training of deep bidirectional transformers for language understanding. arXiv:1810.04805.

Dore, K. M., Hansen, M. F., Klegarth, A. R., Fichtel, C., Koch, F., Springer, A., Kappeler, P., Parga, J. A., Humle, T., Colin, C., Raballand, E., Huang, Z.-P., Qi, X.-G., Di Fiore, A., Link, A., Stevenson, P. R., Stark, D. J., Tan, N., Gallagher, C. A., Anderson, C. J., Campbell, C. J., Kenyon, M., Pebsworth, P., Sprague, D., Jones-Engel, L., and Fuentes, A. (2020). Review of GPS collar deployments and performance on nonhuman primates. Primates.

Farine, D. R. and Whitehead, H. (2015). Constructing, conducting and interpreting animal social network analysis. Journal of Animal Ecology, 84(5):1144-1163.

Fehlmann, G. and King, A. J. (2016). Bio-logging. Current Biology, 26(18):R830-R831.

Furuichi, T. (1984). Symmetrical patterns in non-agonistic social interactions found in unprovisioned Japanese macaques. Journal of Ethology, 2(2):109-119.

Gelardi, V., Godard, J., Paleressompoulle, D., Claidiere, N., and Barrat, A. (2020). Measuring social networks in primates: wearable sensors versus direct observations. Proceedings of the Royal Society A: Mathematical, Physical and Engineering Sciences, 476(2236):20190737.

Haddadi, H., King, A. J., Wills, A. P., Fay, D., Lowe, J., Morton, A. J., Hailes, S., and Wilson, A. M. (2011). Determining association networks in social animals: choosing spatial-temporal criteria and sampling rates. Behavioral Ecology and Sociobiology, 65(8):1659-1668.

Heupel, M. R., Semmens, J. M., and Hobday, A. J. (2006). Automated acoustic tracking of aquatic animals: scales, design and deployment of listening station arrays. Marine and Freshwater Research, 57(1):1-13.

Hornik, K. (1991). Approximation capabilities of multilayer feedforward networks. Neural Networks, $4(2): 251-257$.

Jin, L., Gupta, M. M., and Nikiforuk, P. N. (1995). Universal approximation using dynamic recurrent neural networks: discrete-time version. In Proceedings of ICNN'95 - International Conference on Neural Networks, volume 1, pages 403-408.

King, A. J., Clark, F. E., and Cowlishaw, G. (2011a). The dining etiquette of desert baboons: the roles of social bonds, kinship, and dominance in co-feeding networks. American Journal of Primatology, 73(8):768774. 
King, A. J., Fehlmann, G., Biro, D., Ward, A. J., and Fürtbauer, I. (2018). Re-wilding collective behaviour: An ecological perspective. Trends in Ecology $\&$ Evolution, 33(5):347-357.

King, A. J., Sueur, C., Huchard, E., and Cowlishaw, G. (2011b). A rule-of-thumb based on social affiliation explains collective movements in desert baboons. Animal Behaviour, 82(6):1337-1345.

Krause, J., James, R., Franks, D. W., and Croft, D. P., editors (2015). Animal Social Networks. Oxford University Press.

Levinson, S. C. (2016). Turn-taking in human communication - origins and implications for language processing. Trends in Cognitive Sciences, 20(1):6-14.

Maekawa, T., Ohara, K., Zhang, Y., Fukutomi, M., Matsumoto, S., Matsumura, K., Shidara, H., Yamazaki, S. J., Fujisawa, R., Ide, K., Nagaya, N., Yamazaki, K., Koike, S., Miyatake, T., Kimura, K. D., Ogawa, H., Takahashi, S., and Yoda, K. (2020). Deep learning-assisted comparative analysis of animal trajectories with deephl. Nature Communications, 11(1):5316.

Martins, A. and Astudillo, R. (2016). From softmax to sparsemax: A sparse model of attention and multilabel classification. In Balcan, M. F. and Weinberger, K. Q., editors, Proceedings of The 33rd International Conference on Machine Learning, volume 48 of Proceedings of Machine Learning Research, pages 16141623, New York, New York, USA. PMLR.

Mitani, M. (1986). Voiceprint identification and its application to sociological studies of wild Japanese monkeys (Macaca fuscata yakui). Primates, 27(4):397-412.

Morita, T., Toyoda, A., Aisu, S., Kaneko, A., Suda-Hashimoto, N., Matsuda, I., and Koda, H. (2020). Animals exhibit consistent individual differences in their movement: A case study on location trajectories of Japanese macaques. Ecological Informatics, 56:101057.

Nakagawa, N., Nakamichi, M., and Sugiura, H. (2010). The Japanese Macaques. Primatology Monographs. Springer Japan.

Niculae, V. and Blondel, M. (2017). A regularized framework for sparse and structured neural attention. In Guyon, I., Luxburg, U. V., Bengio, S., Wallach, H., Fergus, R., Vishwanathan, S., and Garnett, R., editors, Advances in Neural Information Processing Systems 30, pages 3338-3348. Curran Associates, Inc.

Niculae, V., Martins, A., Blondel, M., and Cardie, C. (2018). SparseMAP: Differentiable sparse structured inference. In Dy, J. and Krause, A., editors, Proceedings of the 35th International Conference on Machine 
Learning, volume 80 of Proceedings of Machine Learning Research, pages 3799-3808, Stockholmsmässan, Stockholm Sweden. PMLR.

Otani, Y., Sawada, A., and Hanya, G. (2014). Short-term separation from groups by male Japanese macaques: Costs and benefits in feeding behavior and social interaction. American Journal of Primatology, 76(4):374384.

Pays, O., Benhamou, S., Helder, R., and Gerard, J.-F. (2007). The dynamics of group formation in large mammalian herbivores: an analysis in the european roe deer. Animal Behaviour, 74(5):1429-1441.

Pérez, J., Marinkovic, J., and Barceló, P. (2019). On the turing completeness of modern neural network architectures. In 7th International Conference on Learning Representations, ICLR 2019, New Orleans, LA, USA, May 6-9, 2019.

Primate Research Institute (2010). The Care and Use of Laboratory Primates. The Primate Research Institute, Kyoto University, third edition.

Rutz, C., Burns, Z. T., James, R., Ismar, S. M. H., Burt, J., Otis, B., Bowen, J., and St Clair, J. J. H. (2012). Automated mapping of social networks in wild birds. Current Biology, 22(17):R669-R671.

Rutz, C. and Hays, G. C. (2009). New frontiers in biologging science. Biology Letters, 5(3):289-292.

Schofield, D., Nagrani, A., Zisserman, A., Hayashi, M., Matsuzawa, T., Biro, D., and Carvalho, S. (2019). Chimpanzee face recognition from videos in the wild using deep learning. Science Advances, 5(9).

Shaw, P., Uszkoreit, J., and Vaswani, A. (2018). Self-attention with relative position representations. In Proceedings of the 2018 Conference of the North American Chapter of the Association for Computational Linguistics: Human Language Technologies, Volume 2 (Short Papers), pages 464-468, New Orleans, Louisiana. Association for Computational Linguistics.

Silk, J., Alberts, S., and Altmann, J. (2006a). Social relationships among adult female baboons (Papio cynocephalus) II. variation in the quality and stability of social bonds. Behavioral Ecology and Sociobiology, $61: 197-204$.

Silk, J., Altmann, J., and Alberts, S. (2006b). Social relationships among adult female baboons (Papio cynocephalus) I. variation in the strength of social bonds. Behavioral Ecology and Sociobiology, 61:183195.

Sosa, S., Sueur, C., and Puga-Gonzalez, I. (2020). Network measures in animal social network analysis: Their strengths, limits, interpretations and uses. Methods in Ecology and Evolution. 
Sun, J., Taylor, D., and Bollt, E. M. (2015). Causal network inference by optimal causation entropy. SIAM Journal on Applied Dynamical Systems, 14(1):73-106.

Takeda, R. and Komatani, K. (2016). Sound source localization based on deep neural networks with directional activate function exploiting phase information. In 2016 IEEE International Conference on Acoustics, Speech and Signal Processing (ICASSP), pages 405-409.

van den Oord, A., Dieleman, S., Zen, H., Simonyan, K., Vinyals, O., Graves, A., Kalchbrenner, N., Senior, A., and Kavukcuoglu, K. (2016a). Wavenet: A generative model for raw audio.

van den Oord, A., Kalchbrenner, N., Espeholt, L., kavukcuoglu, k., Vinyals, O., and Graves, A. (2016b). Conditional image generation with PixelCNN decoders. In Lee, D. D., Sugiyama, M., Luxburg, U. V., Guyon, I., and Garnett, R., editors, Advances in Neural Information Processing Systems 29, pages 47904798. Curran Associates, Inc.

Vaswani, A., Shazeer, N., Parmar, N., Uszkoreit, J., Jones, L., Gomez, A. N., Kaiser, L. u., and Polosukhin, I. (2017). Attention is all you need. In Guyon, I., Luxburg, U. V., Bengio, S., Wallach, H., Fergus, R., Vishwanathan, S., and Garnett, R., editors, Advances in Neural Information Processing Systems 30, pages 5998-6008. Curran Associates, Inc.

Yamada, M. (1963). A study of blood-relationship in the natural society of the Japanese macaque. Primates, $4(3): 43-65$.

Yuan, Y. and Jia, K. (2019). FusionAtt: Deep fusional attention networks for multi-channel biomedical signals. Sensors, 19(11):2429. 